\title{
Signal-Processing and Adaptive Prototissue Formation in Metabolic DNA Protocells
}

Avik Samanta, ${ }^{1 *}$ Maximilian Hörner, ${ }^{3}$ Wei Liu ${ }^{1}$, Wilfried Weber, ${ }^{3}$ Andreas Walther ${ }^{1-2 *}$

${ }^{1}$ A3BMS Lab, University of Mainz, Department of Chemistry, Duesbergweg 10-14, 55128 Mainz, Germany.

${ }^{2}$ Cluster of Excellence livMatS @ FIT - Freiburg Center for Interactive Materials and Bioinspired Technologies, University of Freiburg, Freiburg, Germany

${ }^{3}$ Faculty of Biology, Signalling Research Centres BIOSS and CIBSS, University of Freiburg, 79104 Freiburg, Germany.

Email: avik.samanta@uni-mainz.de, andreas.walther@uni-mainz.de 


\section{Abstract}

The fundamental life-defining processes in living cells, such as replication, division, adaptation, and tissue formation, take place via intertwined metabolic reaction networks orchestrating downstream signal processing in a confined, crowded environment with high precision. Hence, it is crucial to understand and reenact some of these functions in wholly synthetic cell-like entities (protocells) to envision designing soft-materials with life-like traits. Herein, we report on a programmable all-DNA protocell (PC) composed of a liquid DNA interior and a hydrogel-like shell, harboring DNAzyme active sites in the interior whose catalytic bond-cleaving activity leads to a downstream phenotype change in the protocells, as well as triggers prototissue formation. In this regard, we coupled several tools of DNA nanoscience, such as RNA cleavage, dynamic strand displacement reactions, and multivalent palindromic interactions, in a synchronize pathway so that the input signal can be processed inside the protocells and generate downstream cues giving rise to metabolic adaptive behavior. For example, the compartmentalized DNAzyme catalyzes the bond-cleavage of a substrate that releases a DNA strand in situ to trigger a strand displacement reaction at the shell of the protocells leading to a change in color resembling a "phenotype-like" change in cells, and finally to establish communication with other protocells via multivalent interactions. 
iving cells execute numerous complex functions, such as division, differentiation, adaptation, and tissue formation, by guiding the flux of materials and information via intertwined metabolic reaction networks in a crowded intracellular environment or even in liquid/liquid phase-segregated (LLPS) membraneless organelles..$^{1-5}$ In recent years, the bottom-up reenactment of fundamental cellular processes inside artificial synthetic compartments has emerged as an appealing strategy to investigate biological pathways from molecular to macroscopic length-scale in a controlled environment. ${ }^{6-9}$ These approaches not only pave the way towards constructing a wholly man-made synthetic cellular equivalent (Protocells, PCs) with life-like functionality and adaptivity but also provide an insight into fundamental reaction network-driven processes in natural cells. Historically, liposomes, ${ }^{10-15}$ polymersomes, ${ }^{16-18}$ colloidosomes, ${ }^{19,}{ }^{20}$, and proteinosomes ${ }^{21}$ based PC models have been used for encapsulation of bio-inspired transformations or also communication networks. This involves, for instance, DNA-mediated signal cascades, self-replication, and in-vitro protein synthesis. ${ }^{22}$ However, such systems may suffer from low and heterogeneous encapsulation efficiency, absence of molecularly crowded interior resembling the cytoplasmic matrix, and lack control over stoichiometry of components in the case of multimeric active cargoes. In contrast, protocell models ${ }^{23-26}$ based upon complex coacervates, aqueous twophase systems, and LLPS of intrinsically disordered proteins have been suggested as more appropriate cytoplasmic model systems because their inherent macromolecular-rich crowded interior and high loading capacity resemble the cytoplasmic matrix. ${ }^{27-30}$ Various studies reported the use of such macromolecularly crowded PCs for gene expression, ${ }^{31}$ ribonucleic acid catalysis ${ }^{32}, 33$, and multienzyme iterative processing in multicomponent microdroplets. ${ }^{34,35}$ In spite of these recent examples, functional adaptation in crowded PCs using metabolic transformations based upon an external signal using an encapsulated catalyst with precisely organized downstream action remain challenging and are rarely explored. ${ }^{19}$ Recently, we reported the functional and morphological adaptation ability of all-DNA PCs ${ }^{36}$ via in-situ generation of a self-reporting nonDNA metabolite upon ring-closing metathesis reaction based on genetically modified artificial metalloenzymes immobilized in PCs. ${ }^{37}$ The non-DNA metabolite could interact with double-stranded DNA (dsDNA) in the shell, which led to metabolic growth, mechanical stress built up, and ultimately PC fusion. Since adaptivity is imperative for life's survival in a dynamic environment, it is of critical importance to establish strategies capable of converting chemical signals from diverse origins to allow for intra- or inter-PC downstream processes such as functional adaptation and communication.

DNA has become a relevant biomacromolecule for nanoscience and systems chemistry research for its programmable structure formation and its ability to rationally design reaction networks and concatenated logic operations, demonstrating its potential as a toolbox in molecular computing. ${ }^{38-44}$ Among other DNA principles, catalytic nucleic acids (DNAzymes and ribozymes) ${ }^{45-48}$ have emerged as one relevant tool in the DNA reaction network toolbox. Towards a first combination of DNAzymes with PCs, recently, it has been shown that the ribozyme activity of bond cleavage is enhanced inside membraneless coacervate droplets. ${ }^{33}$ However, a higherlevel function integration of such systems in protocell research remains elusive. 
Herein we introduce catalytic signal processing inside highly programmable all-DNA protocells (PC) formed by LLPS of specific single-stranded DNA (ssDNA) strands. ${ }^{36,37,49}$ As a key tool, we encapsulate a DNAzyme able to cleave RNA positions in DNA-RNA chimera substrates that serve as signals. We demonstrate efficient metabolic signal conversion by RNA bond cleavage and capitalize on this understanding by using the signal output to instigate downstream "phenotype changes" in the PC, as well as of prototissue formation by PC clustering via induction of multivalent self-complementary interaction at the PC surface. These strategies are enabled by engaging the metabolized output signal in downstream dynamic DNA strand displacement (DSD) reactions.

Building upon our previous work, ${ }^{36,37,49}$ we prepared PCs composed of liquid ssDNA interiors and DNA hydrogel shells by rapid self-compartmentalization during a temperature ramp (ca. $10 \mathrm{~min}$ ) of an aqueous solution containing two ssDNA-multiblock copolymers $\left(p\left(A_{20}-m\right)\right.$ and $\left.p\left(T_{20}-n\right)\right)$. $A_{20}$ and $T_{20}$ denote homo-repeats of 20 adenine and 20 thymine nucleotides (nt), while $\mathrm{m}$ and $\mathrm{n}$ stand for defined, so-called barcode domains, which are used for functionalization using their complementary counterparts (Figure 1a). In short, during heating, the $A_{20} / T_{20}$ duplexes dissociate, and $p\left(A_{20}-m\right)$ undergoes LLPS during the heating step, while $p\left(T_{20}-n\right)$ remains dissolved. During cooling, re-hybridization of $A_{20} / T_{20}$ happens rapidly at the periphery of the $p\left(A_{20}-m\right)$ phaseseparated droplets, giving rise to a hydrogel-like shell stabilized by $A_{20} / T_{20}$ duplexes. Eventually, the $p\left(A_{20}-m\right)$ dissolves below its cloud point temperature $\left(\mathrm{ca} .45^{\circ} \mathrm{C}\right),{ }^{36}$ but stays entrapped in a liquid state under high osmotic pressure and macromolecular crowding, as confined by a compact hydrogel-like shell. Apart from the facile preparation and providing a cytosol-like DNA-based crowded, confined platform, one of the significant advantages of such PCs is the selective addressability and orthogonal functionalization of the interior and the shell using the barcode domains $(m, n)$ (Supplementary Figure S2, Table S1).

To realize the catalytic metabolism inside the PCs, we introduced a specific $\mathrm{Mg}^{2+}$-dependent RNA-cleaving DNAzyme (Dz). This specific DNAzyme cleaves a single RNA linkage embedded in a DNA substrate efficiently. ${ }^{47}$ For the facile loading of $\mathrm{Dz}$ in the PC core, we separated it into two ssDNA sequences, so-called split DNAzymes (Figure 1a, 2a, and Supplementary Figure S1 ). Each sequence contains one-half of the complementary barcode domain $\left(\mathrm{m}_{1 / 2}{ }^{*}\right)$, a stem part (s or $\left.\mathrm{s}^{*}\right)$, an active site sequence $\left(\mathrm{t}_{1}\right.$ or $\left.\mathrm{t}_{2}\right)$, and a substrate docking site $\left(\mathrm{u}_{1}\right.$ or $\left.\mathrm{u}_{2}\right)$. After annealing both parts to form the acting Dz, these Dzs were added to a buffered PC dispersion containing $50 \mathrm{mM} \mathrm{Mg}^{2+}$ (for all the experiments hereafter), whereupon the Dzs rapidly dock inside the PC core via binding with every $m$ barcode sequence present in the $p\left(A_{20}-m\right)$ chains. Since the concentration of $m$-barcode in the PC interior can be precisely determined, all the barcodes can be easily functionalized with Dzs using proper amounts added to the dispersion. Any excess $\mathrm{Dz}$ in solution is removed by washing the PCs via centrifugation.

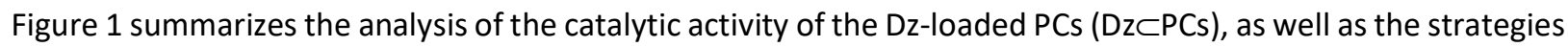
for downstream signal processing in a step-by-step manner using three different thoughtfully designed substrates (Figure 1b): (1) A self-reporting substrate (Subs-1) with an RNA linkage, in which a fluorescence resonance energy transfer (FRET) pair (fluorophore+quencher) is separated upon bond-cleavage helps to analyze the catalytic conversion. (2) Subs-3 with a cleavage domain at its loop (Supplementary Figure S3)., 
concealing a sequence $\left(n^{*}\right)$ complementary to the shell barcode that is released upon catalytic loop cleavage, is used for the phenotype change by DSD of shell-immobilized shorter Atto $488-\mathrm{n}_{\text {short }}{ }^{*}$. (3) A loop substrate Subs4 with a palindromic sequence connected to $n^{*}(C y 5-n *-p)$ is used to prompt inter-PC communication by multivalent binding scenarios.

a. Assembly of all-DNA protocell and DNAzyme loading

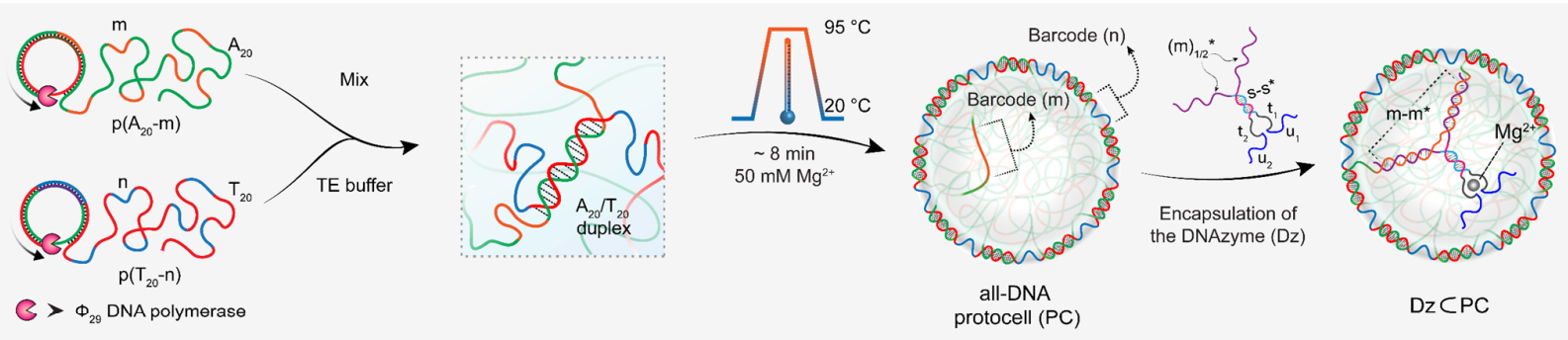

b. System Behavior

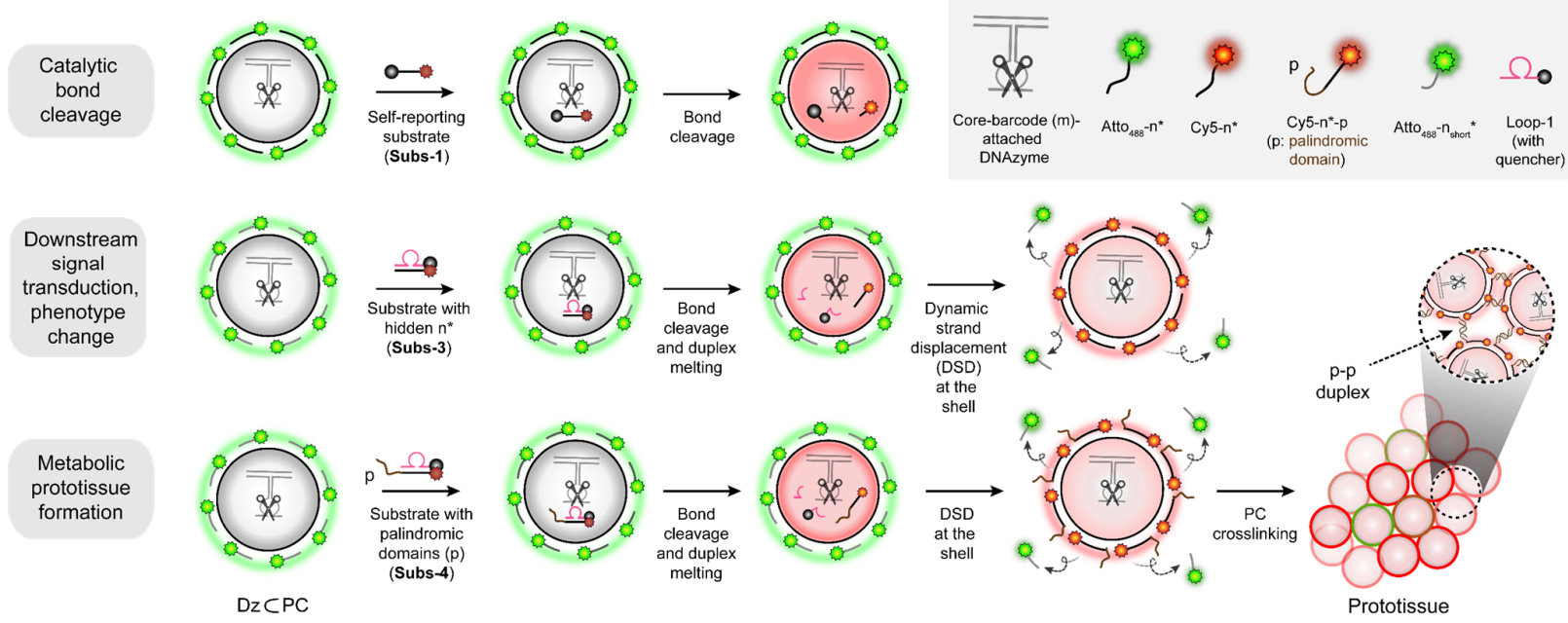

Figure 1. Design, strategy, and system behavior of DNAzyme-catalyzed signal conversation, downstream functional adaptation inside all-DNA PCs, and metabolic prototissue formation. a) Synthesis of sequence-specific two multiblock ssDNA polymers via rolling circle amplification (RCA). A buffered mixture of the ssDNA polymers is subjected to a fastheating ramp $\left(3^{\circ} \mathrm{C} / \mathrm{min}\right)$ in the presence of $\mathrm{Mg}^{2+}(50 \mathrm{mM})$. Heat-induced phase-separation of $\mathrm{p}\left(\mathrm{A}_{20}-\mathrm{m}\right)$ during heating and antagonistic duplex $\left(A_{20}-T_{20}\right)$ hybridization (at the coacervate surface) during the cooling step, resulting in the self-assembly of kinetically-trapped all-DNA PCs. The core and shell barcodes are denoted with $\mathrm{m}$ and $\mathrm{n}$, respectively. The immobilization of DNAzymes $(\mathrm{Dz})$ is achieved using duplex hybridization between $\mathrm{m}^{*}$ residues of the $\mathrm{Dz}$ and the core barcodes $(\mathrm{m})$. $\left.b\right)$ The system properties are demonstrated in three different pathways. Firstly, a self-reporting substrate (Subs-1) carrying an RNA-linkage is used to investigate the catalytic efficiency of Dz in the confined state, in which Cy5 fluorescence (red color) increases upon cleaving the RNA-linkage. Secondly, the downstream signal processing and phenotype change in PCs are demonstrated using a caged substrate (Subs-3). The Dz-catalyzed bond cleavage at the Loop-1 and subsequent release of Cy5-n* substitutes Atto $488-n_{\text {short }}{ }^{*}$, changing the shell color from green to red. Thirdly, the metabolic prototissue formation is demonstrated by a similar loop substrate (Subs-4) bearing a palindromic ( $p$ ) sequence, which gets exposed at the PC periphery after the intra-protocellular metabolic bond cleavage and a DSD reaction at the shell, leading to crosslinking of the PCs via p-p duplex formation. All the strands are listed in Supplementary Table S2.

Firstly, to investigate the catalytic activity of the Dz inside and outside the PCs, we used a self-reporting substrate (Subs-1) with an RNA linkage and a FRET pair (Cy5 and a black hole quencher (BHQ)) covalently attached at both ends. Upon Dz-catalyzed breaking of the RNA bond in Subs-1, Cy5 and BHQ are separated (Figure 2a), giving rise to the enhancement of fluorescence intensity (represented in red color). The stoichiometric ratio of Subs-1 concentration to Dz concentration is fixed at 2.5 for comparing the catalyst activity. In more detail, Subs- 1 binds 
to the $u_{1}$ and $u_{2}$ residues of the $D z$, but after cleavage, the two parts of the initial ssDNA signal melt away due to the low melting temperature $\left(T_{\mathrm{m}}=\sim 20^{\circ} \mathrm{C}\right)$ of each individual half. The corresponding fluorescence traces show a 2-fold more efficient Dz-catalyzed bond cleavage (DCBC) in the PC as compared to the solution, also featuring a slightly faster reaction rate (Figure $2 \mathrm{c}$ ). The negative control shows almost no product formation in the absence of the Dz. The percentage of DCBC is close to 62\% in Subs-1 (turnover number 1.6) for the DzCPCs $\mathrm{Dz}$ inside the $\mathrm{PC}$ with respect to the in solution, which is only around $\sim 35 \%$ at the equivalent catalyst loading. We also observe that the encapsulated Dz in PC loses its activity beyond this ratio, which indicates that the cleaved product reaches an equilibrium between free and bound state for its stickiness to the catalytic site at $25^{\circ} \mathrm{C}$ (Supplementary Figure S4).

To achieve more detailed insights on the level of the PC population, we performed flow cytometry experiments.

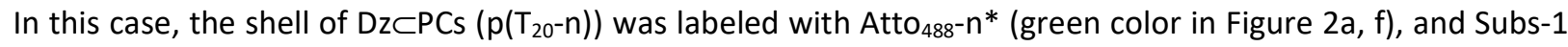
was added after $\sim 2$ min of measurement. PCs were identified in the forward scatter (FSC, size) versus side scatter (SSC, complexity/granularity) plot, and signals without Atto488 and Cy5 fluorescence were excluded from the analysis ( $<3 \%$ of all protocells, Figure $2 \mathrm{e}$ ). The fluorescence intensity in the Cy 5 channel increases after the addition of Subs-1 and reaches a plateau in $350 \mathrm{~s}$ after Subs-1 addition (Figure $2 \mathrm{~g}$ ). This corresponds to the Dzcatalyzed cleavage of Subs-1. In contrast, the Atto ${ }_{488}$ fluorescence of the shell remained unaltered over the measurement window (Figure 2f). The consistent evolution of the cytometry data underscores a homogeneous PC population. The flow cytometry experiments with dormant PCs (without the whole Dz) do not show a steady increase in fluorescence at the Cy5 channel (Supplementary Figure S5).

Furthermore, we selected the Atto 488 -labeled DzCPCs for in-situ microscopy studies using confocal laser scanning microscopy (CLSM), which are initially visualized with a green shell and empty core ( $t=0 \mathrm{~min}$, Figure 2h). Once Subs-1 is injected into the PC suspension, a red fluorescence (excitation $637 \mathrm{~nm}$ ) appears in the core of the DzCPCs, which confirms that the compartmentalized DNAzyme catalyzes the RNA bond cleavage, producing the Cy5-appended output. The rate of product formation correlates with both fluorescence spectroscopy and the cytometry results (Figure 2c, g). Interestingly, the red fluorescence persists in the PC core after the completion of the reaction, and an immediate diffusive leveling into the solution does not occur at 25 ${ }^{\circ} \mathrm{C}$, which is also supported by the cytometry results (Figure $2 \mathrm{~g}$ ). The line segment analysis of the confocal micrographs before the substrate addition ( $0 \mathrm{~min}$, line-1), after the addition ( $1 \mathrm{~min}$, Line-2), and at the reaction completion (8 min, Line-3) are shown in Figure 2i-k, respectively, exhibiting an increase of red fluorescence along the lines throughout the reaction. In contrast, the green fluorescence at the shell remains unchanged. In a relevant control experiment, no Cy5 fluorescence enhancement is observed in the PC core when the same amount of Subs-1 was injected into a dormant PC (without the encapsulated Dz; Figure 2I). 


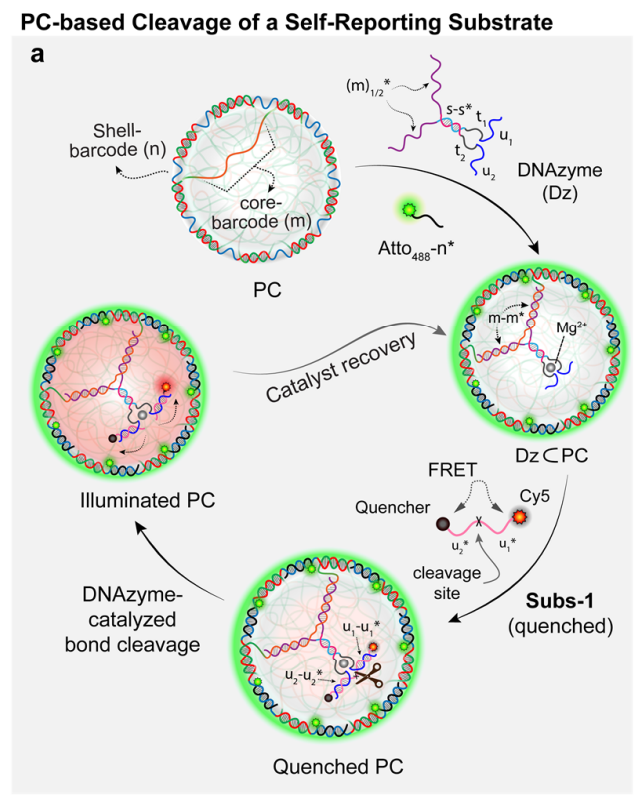

DNAzyme Efficiency in Solution and in PC

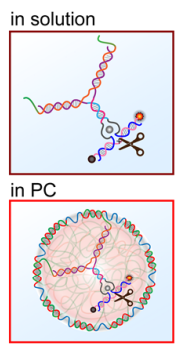

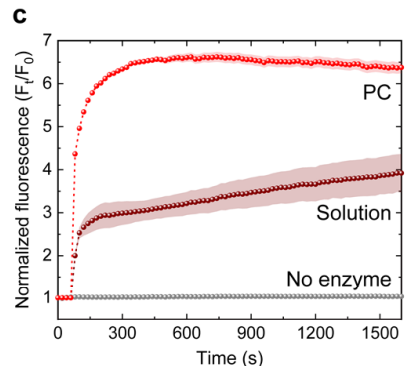

d

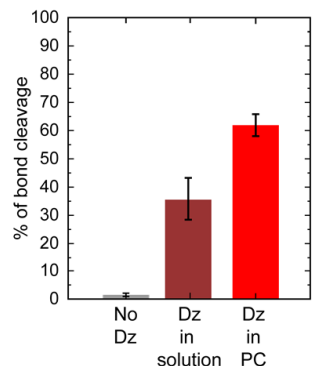

Flow Cytometry Analysis of DNAzyme-Catalyzed Bond Cleavage in PC

e
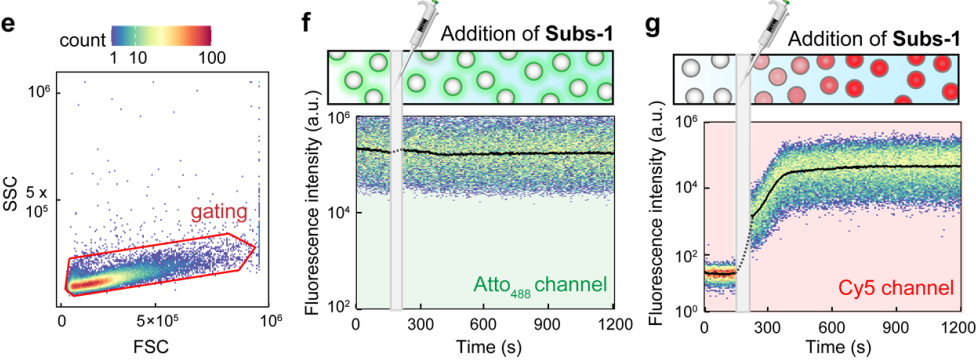

Monitoring the Cleavage of Subs-1 via CLSM Imaging

h
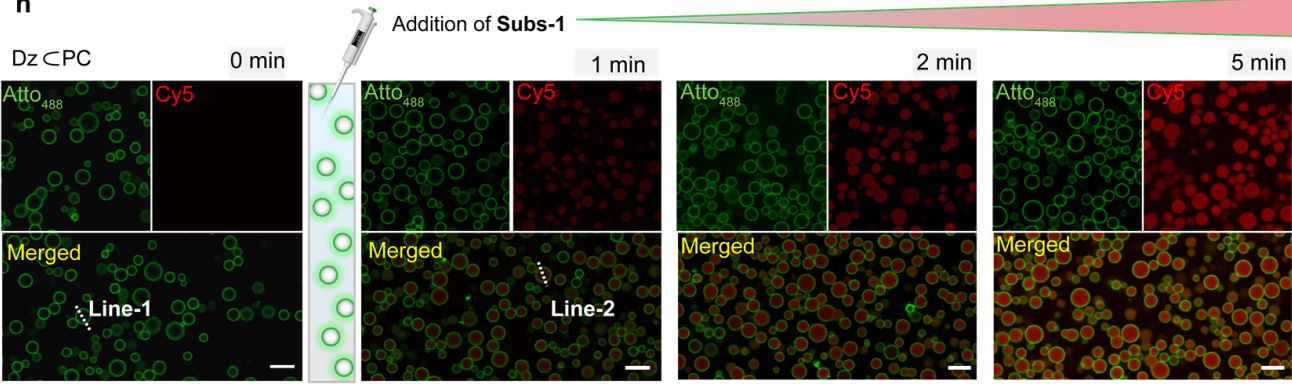

Reaction progress
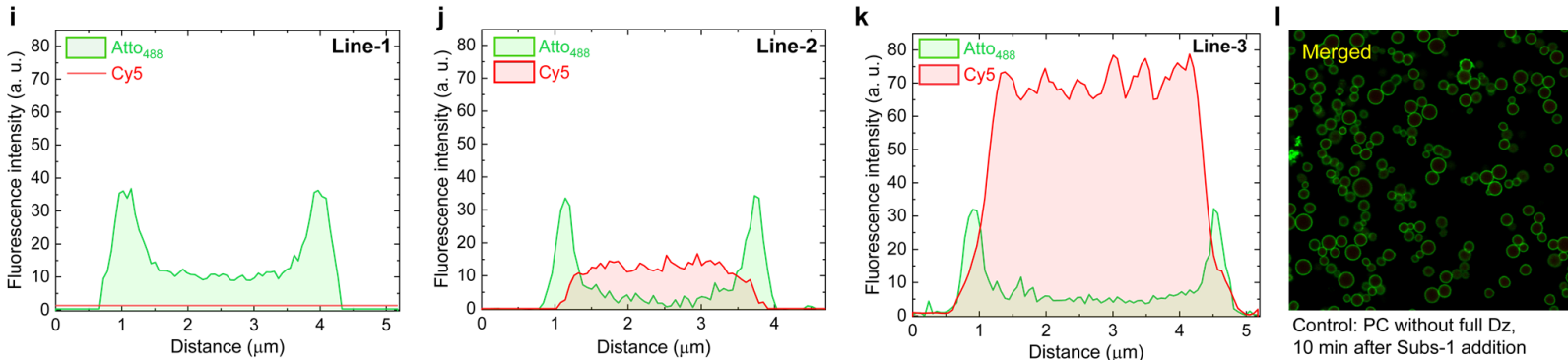

Figure 2. Intra-protocellular catalytic cleavage of self-reporting substrate. a) Schematic representation of a catalytic cycle for RNA-linkage cleavage in Subs-1 in PC interior and fluorescence enhancement upon uncaging of the Cy5-appended product. b) Schematic representation of two scenarios: i) the DZ-catalyzed bond cleavage (DCBC) is performed in a buffered solution where the $\mathrm{Dz}$ is hybridized with a monomeric m sequence and ii) DCBC is performed in DzCPCs. c) Time-dependent spectrofluorimetric investigation of DCBC in PC and in solution. A control experiment in which the auto-cleavage of Subs-1 is also presented in the absence of Dz (grey trace). d) The percentage of bond cleavage of Subs-1 in the three scenarios mentioned above. e) PCs were identified in the forward scatter (FSC) versus side scatter (SSC) plot. f) and g) Intraprotocellular Atto 488 and $\mathrm{Cy} 5$ fluorescence was monitored over time by flow cytometry. Black line shows the moving median. h) The time-dependent CLSM images for DCBC induced Cy5-appended sequence uncaging in a Atto 488 -labeled PC system. The shell of all the PCs is labeled with Atto $488-n *$ (green channel). At $t=0$ (before the addition of Subs- 1 ), all the PCs are visualized as green circles since the fluorescent Atto $488-n *$ is hybridized with the shell barcode domain $(n)$ of $p\left(T_{20}-n\right)$ polymer. The DCBC in DzCPCs is monitored over $8 \mathrm{~min}$ time period, and a gradual increase of Cy5 fluorescence (red channel) at the PC core is observed. i), j), and k) Line segment analysis in both channels (green and red) of Line- 1 , Line-2 and Line-3 at $t=0,1$ and $8 \mathrm{~min}$, respectively. I) CLSM image of dormant PCs (without the Dz, only with Split-Dz-1) at $\mathrm{t}=10 \mathrm{~min}$ after the addition of Subs-1. Data are the means \pm standard deviation of duplicate reactions. Scale bars: $5 \mu \mathrm{m}$. Condition: for 


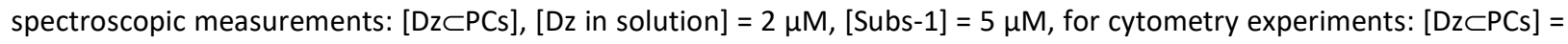
$0.5 \mu \mathrm{M},\left[\right.$ Subs-1] $=1.3 \mu \mathrm{M}$ and for the microscopic experiments: [DzCPCs] $=7 \mu \mathrm{M},\left[\right.$ Subs-1] $=16.6 \mu \mathrm{M}$, at $25^{\circ} \mathrm{C}, \mathrm{TE}$ buffer at $\mathrm{pH} 8,50 \mathrm{mM} \mathrm{MgCl}_{2}$.

To better understand the diffusivity of the output product of the DCBC and the potential binding to the substrate recognition site (u1, u2), we performed FRAP (Fluorescence Recovery After Photobleaching) cycles inside the DzCPC interior. The repeated photobleaching on a PC after completion of the reaction (cleaving the Subs-1) in both Atto $_{488}$ (green) and Cy5 (red) channels exhibits different diffusion patterns. The green fluorescence at the PC shell (region of interest $1, \mathrm{ROI} 1$ ) does not show any recovery after every bleach cycle and attains a fully bleached state after the fifth cycle (Figure 3a, b). This corresponds to a hydrogel-like shell structure with negligible fluorophore diffusion. In contrast, the red fluorescence at the PC core (ROI 3) exhibits almost complete recovery after each bleach cycle, confirming rapid diffusion of the product inside the PC core but for some remaining stickiness to the binding site (Figure 3a, c). Note that a control area outside of the PC (ROI 4) also shows bleaching of Cy5-fluorescence and recovery due to some slowly leaked product.

Furthermore, to demonstrate the continued operation and also refreshed use of the encapsulated Dz, we injected a second substrate (Subs-2) with a different fluorophore (Cy3) to the DzCPCs after completion of the Subs-1 cleavage (Figure 3d). Prior to the addition of Subs-2, the PCs with cleaved Subs-1 (with Cy5) were incubated at $37^{\circ} \mathrm{C}$ for $60 \mathrm{~min}$ to foster the release of the cleaved product from the PC core (Figure 3e, f). Before and after incubation, the line segment analysis obtained from CLSM revealed $82 \%$ of product release from the PCs (Figure 3g, h). Upon addition of Subs-2, the Cy3 fluorescence (magenta) increases rapidly inside the PC core, indicating the cleavage of the RNA linkage in Subs-2 (Figure 3i). Figure 3j presents the line segment analysis of the three channels $\left(\mathrm{Atto}_{488}, \mathrm{Cy} 3\right.$, and $\mathrm{Cy} 5$ ) after $8 \mathrm{~min}$ of the Subs-2 addition. These observations corroborate that the Dz, immobilized in the PC core, can be used for multiple catalytic cycles of bond cleavage, which is crucial for a metabolic system. 
a

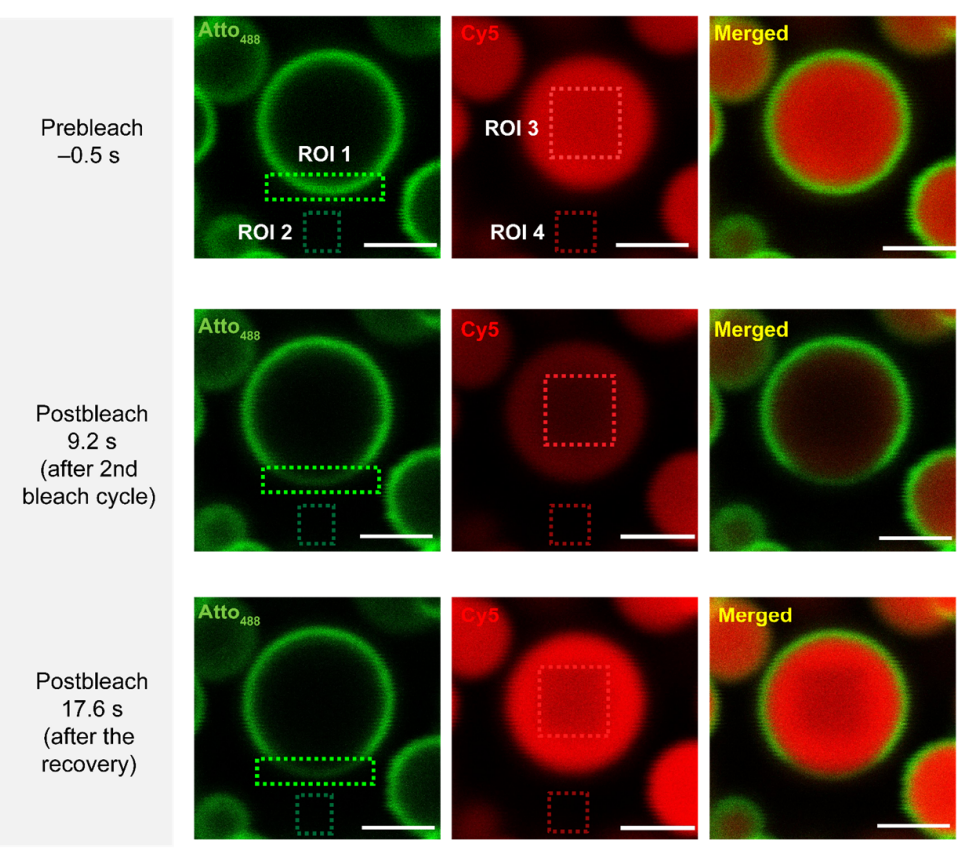

b
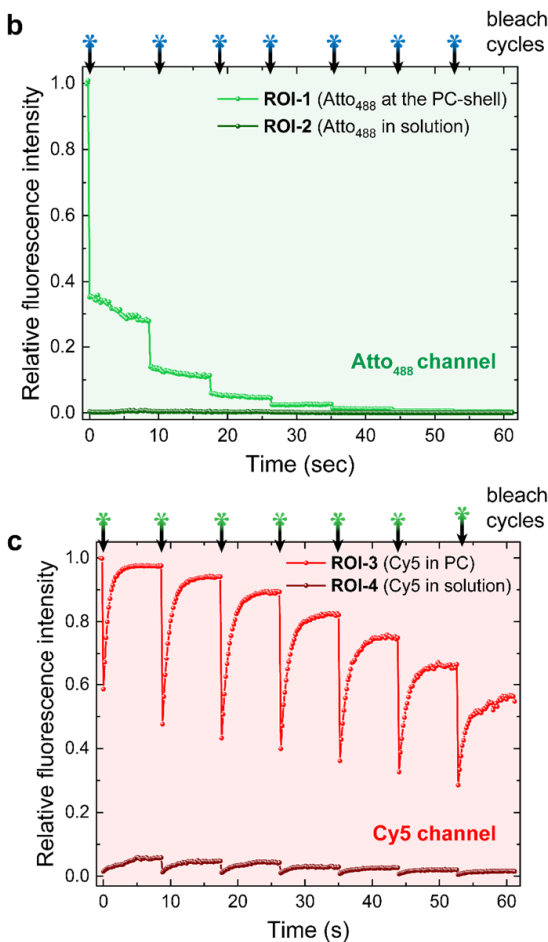

Recovery of the DNAzyme and Cleavage of a Second Substrate

d

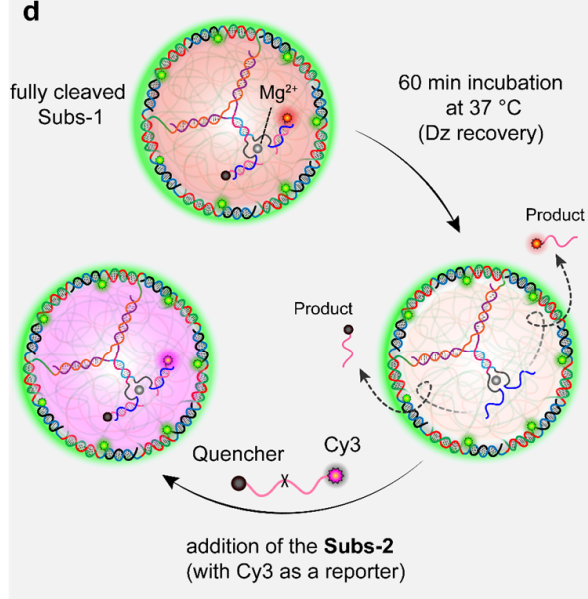

i CLSM Monitoring
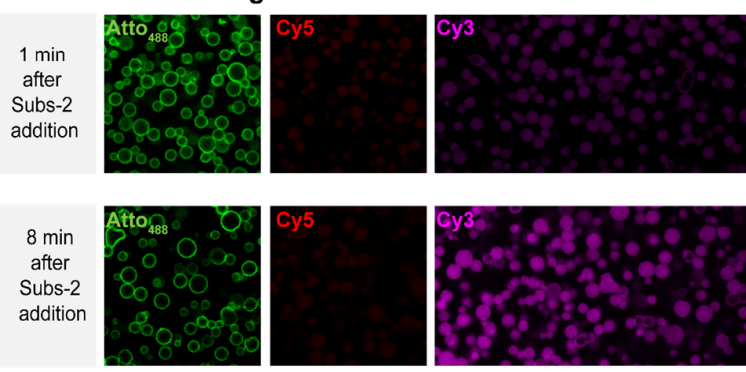

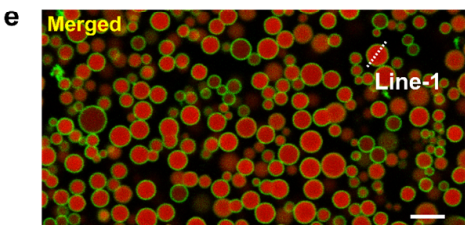

g
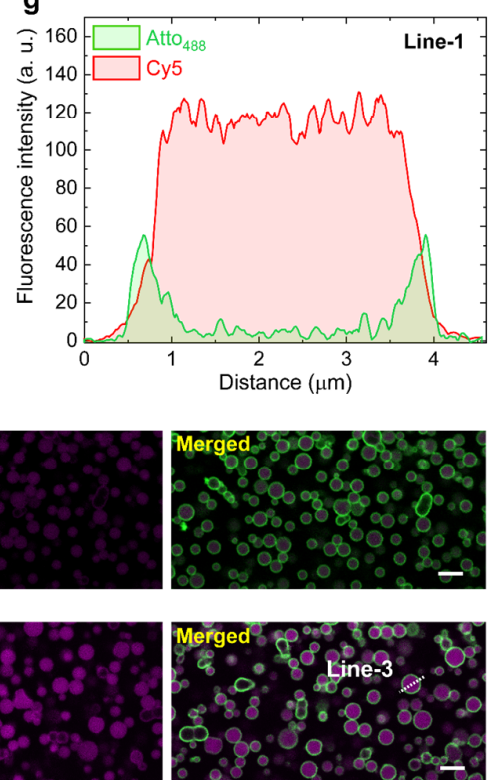

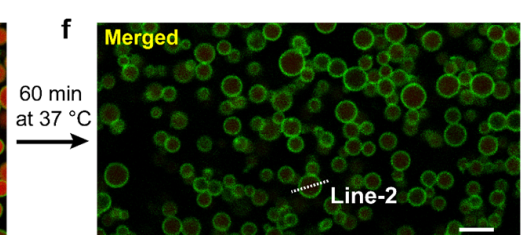

h

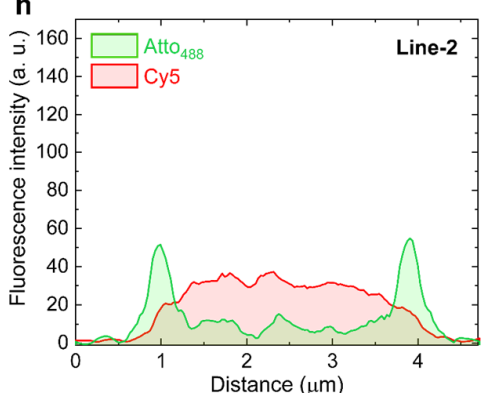

j

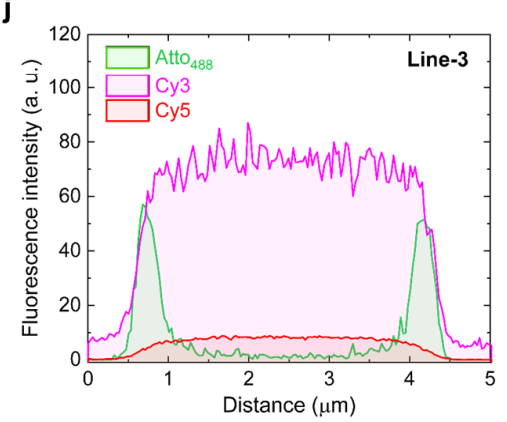

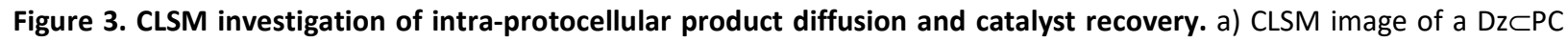
after the completion of DCBC of Subs-1 and the photo-bleaching is performed within the dotted rectangles. Three rows of CLSM images depict the pre-bleach state, the post-bleach state immediately after the second bleach cycle, and another post-bleach state after the recovery, hence just before the third bleaching cycle. b) The relative fluorescence intensities from ROI 3 and ROI 4 (red channel) over the seven bleach cycles. c) The relative fluorescence intensities from ROI 1 and 
ROI 2 (green channel) over seven bleach cycles, depicting Cy5 fluorescence recovery. The black downward arrows represent the bleaching events. Scale bars: $3 \mu \mathrm{m}$. d) Schematic representation of catalyst recovery after completion of the Subs-1 cleavage and the confirmation of the free DNAzyme active sites using Subs-2, a self-reporting substrate with a second fluorophore (Cy3, magenta). e) CLSM image of DzCPCs with fully-cleaved Subs-1 (merged channel: Atto 488 and Cy5). f) CLSM image of DzCPCs after 30 min incubation at $37^{\circ} \mathrm{C}$. g) and h) The line segment analysis in both channels of Line- 1 and Line2 , before and after the incubation, respectively. i) CLSM images Dz $\subset$ PCs after the addition of Subs -2 ( $t=1 \mathrm{~min}$ and $\mathrm{t}=8 \mathrm{~min}$ ). Three channels represent the PC shell (green, Atto 488 ), the residual product of Subs-1 (red, Cy5), and the product generated

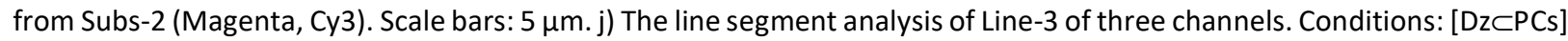
$=5 \mu \mathrm{M},[$ Subs-1] and [Subs-2]= $12.5 \mu \mathrm{M}$ in TE buffer at $\mathrm{pH} 8$.

After investigating the catalytic activity of $\mathrm{Dz}$ and the product diffusion in a confined state, we set out to establish an intra-protocellular downstream adaptation pathway, in which a signal, released via metabolic bond cleavage, triggers a cascade leading to a functional "phenotype" change in the PCs. In this regard, we used a loop substrate (Subs-3), in which a Cy5-appended n* sequence (black) - complementary to the shell barcode of the PC - is concealed using another ssDNA sequence Loop-1 (pink) carrying the RNA linkage at its loop domain and a BHQ at the $3^{\prime}$ end (Figure 4a, b). The Cy5 fluorescence is quenched in Subs-3 because the Cy5 of $\mathrm{n}^{*}$ and the quencher of Loop-1 are in proximity in the hybridized state (Supplementary fig. S1 and S3). Two mismatches were strategically placed at the duplex domains on either side of the loop to keep the $T_{\mathrm{m}}$ around $39^{\circ} \mathrm{C}$ so that both parts of cleaved Loop-1 melt away as soon as the loop is cleaved by the Dz. The loop of Subs3 consists of $11 \mathrm{nt}$ with one RNA linkage at the middle and is designed to be recognized by the $\mathrm{Dz}$ active site. The time-dependent Cy5 fluorescence upon cleavage of the RNA linkage at the loop domain of Subs-3 was monitored via fluorescence spectroscopy after injecting a solution of Subs-3 in three separate scenarios: (i) Dz encapsulated inside the PC (DzCPCs), (ii) Dz are in solution hybridized with monomeric barcode, and (iii) in PCs in a buffered solution without the Dz (Figure 4b, c). Upon addition of Subs-3, the Cy5 fluorescence increases for scenarios (i) and (ii), indicating the DCBC and subsequent bond-cleavage driven duplex melting (BCDM). It is

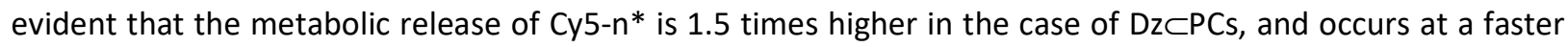
rate than the reaction in solution. This observation corroborates the fact that the catalytic efficiency is higher inside the PCs. The fluorescence intensity increases negligibly in the case of the negative control (no Dz; (iii)), indicating less than $5 \%$ of thermal leakage of Subs-3 at the experimental temperature $\left(37^{\circ} \mathrm{C}\right)$.

To demonstrate the metabolic signal transduction in the PCs, we combined the two consecutive processes, DCBC and BCDM, to release a Cy5-appended ssDNA (Cy5-n*) from Subs-3 inside the DzCPCs, which subsequently diffuses towards the shell and triggers a downstream DSD reaction. The DSD reaction leads to a substitution of the Atto $488-\mathrm{n}_{\text {short }}$ * that was initially present to dye the shell in green, leading thereby to a color change in the PC shell due to tighter anchoring of the Cy5-n* (Figure 4a). This corresponds to a change of the "phenotype" of the PC. The DSD is enabled using an Atto $488-\mathrm{n}_{\text {short }}{ }^{*}$, which only features a complementarity of 13 $\mathrm{nt}$ to the $\mathrm{n}$ barcode domain $(\mathrm{n}=21 \mathrm{nt}$ ), leaving a toehold domain of $8 \mathrm{nt}$ free for the DSD. Flow cytometry analysis of Atto $_{488}-\mathrm{n}_{\text {short }}{ }^{*}$-labeled DzCPC shows a rapid increase in Cy5 fluorescence (red channel) upon injection of Subs-3 (Figure 4e), whereas the fluorescence of Atto 488 (green channel) gradually decreases over time (Figure $4 d$ ). These observations indicate that (i) the Cy5- $n *$ sequence is catalytically released from the input signal (Subs- 
3) upon $D C B C$ and BCDM in the PC core, leading to an enhancement of the red fluorescence, and (ii) that the DSD reaction at the PC shell occurs via ejection of the Atto488- $n_{\text {short }}$ * from the PC shell, resulting in a decrease of the green fluorescence decrease. It is essential to realize that the product is now removed from any substrate/product binding competition at the Dz in the PC interior by spatial relocalization into the shell.

Next, we investigated the Dz-catalyzed downstream signal processing in DzCPCs using in-situ CLSM (Figure 4f$\mathrm{h}$ and Supplementary Figure S6). To clearly monitor changes, we mixed active PCs $\left(\mathrm{AttO}_{488} \mathrm{-}_{\text {short }}{ }^{*}\right.$-labeled DzCPCs; shell in green) with dormant Atto $_{565}-\mathrm{n}_{\text {short }}{ }^{*}$-labeled PCs (without the Dz; shell labeled in magenta; Figure $4 \mathrm{f}, \mathrm{g})$. Upon adding Subs-3 into the mixed PC dispersion, the Cy5 fluorescence (red channel) appears exclusively

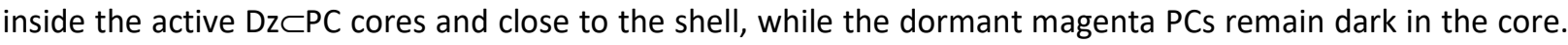

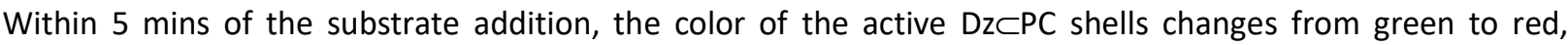
confirming the downstream DSD substitution of $A t \mathrm{tO}_{488}-\mathrm{n}_{\text {short }} *$ in the shell. The Atto $488-\mathrm{n}_{\text {short }} *$ is released into the surroundings and increases the background fluorescence. In contrast, the shell color of the dormant PCs remains unaltered after $8 \mathrm{~min}$, indicating the absence of a metabolic reaction network to produce Cy5- $\mathrm{n}^{*}$. The line

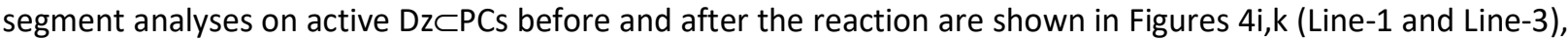
representing the decrease of green fluorescence (Atto ${ }_{488}-n_{\text {short }}{ }^{*}$ ) at the PC shell and enhancement of red fluorescence (Cy5) at the core and mainly at the shell of the active PCs. The intensity of the magenta shell fluorescence of the dormant PCs remains very similar before and after the reaction (Figure 4j,I; Line-2 and Line4). This stability of the magenta fluorescence in the dormant PCs also underscores hardly any diffusive exchange of the Cy5-n* between active and dormant PCs. 
Metabolic Downstream Phenotype Change in PC

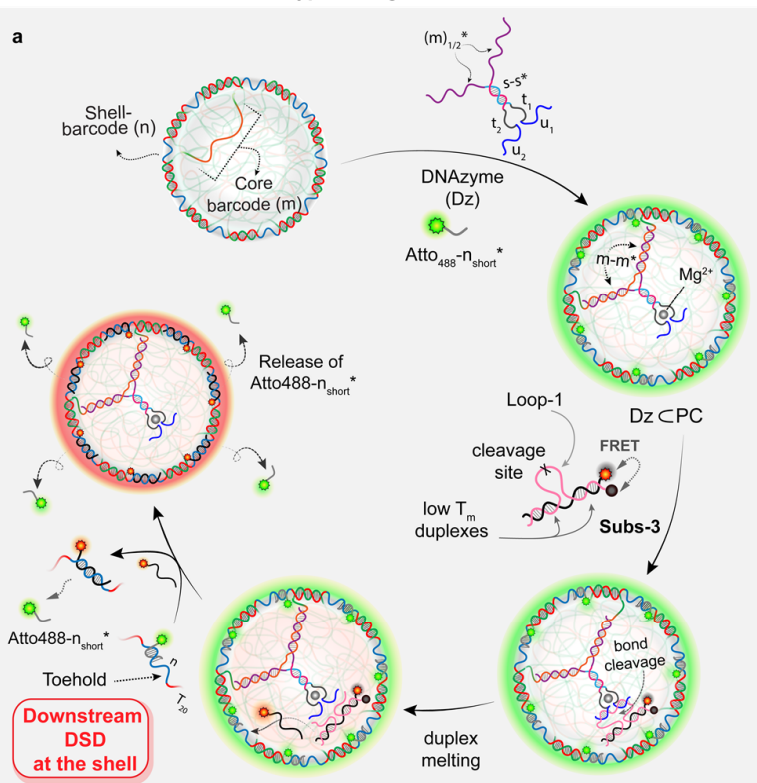

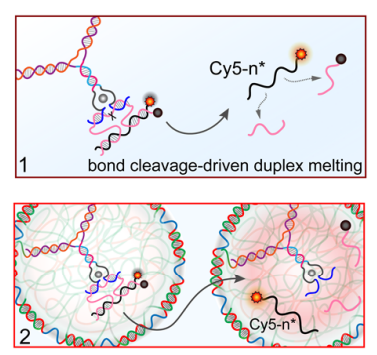

Catalytic Efficiency

c

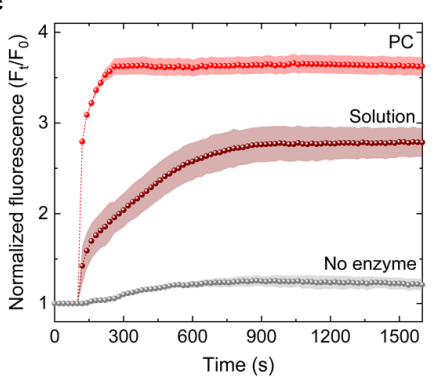

Flow Cytometry Analysis of Downstream Signal Transduction in PC
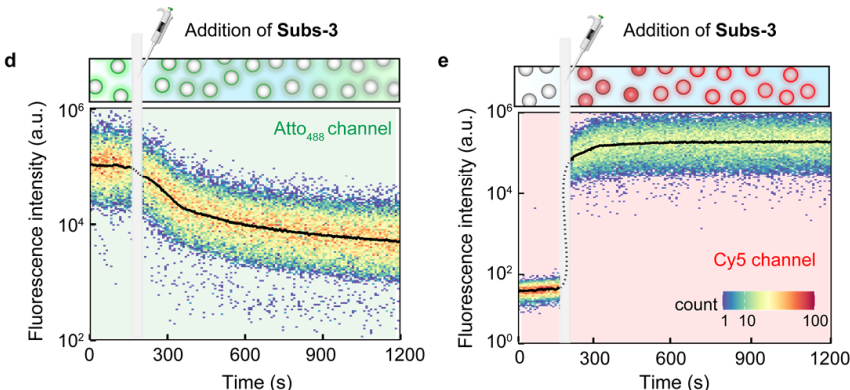

Monitoring of Metabolic Phenotype Change via CLSM Imaging
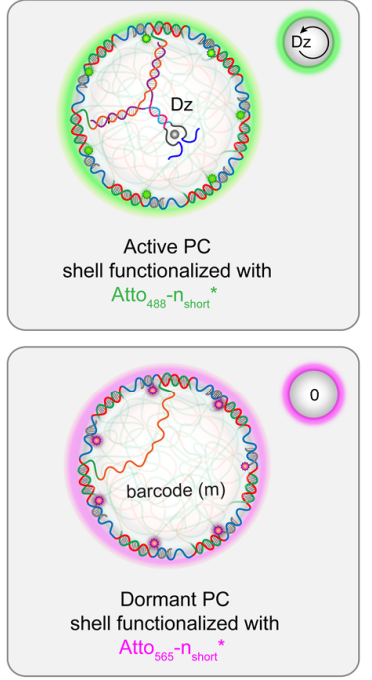

g

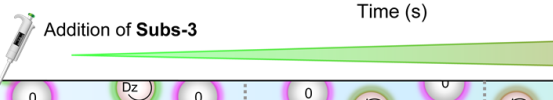

Reaction progress
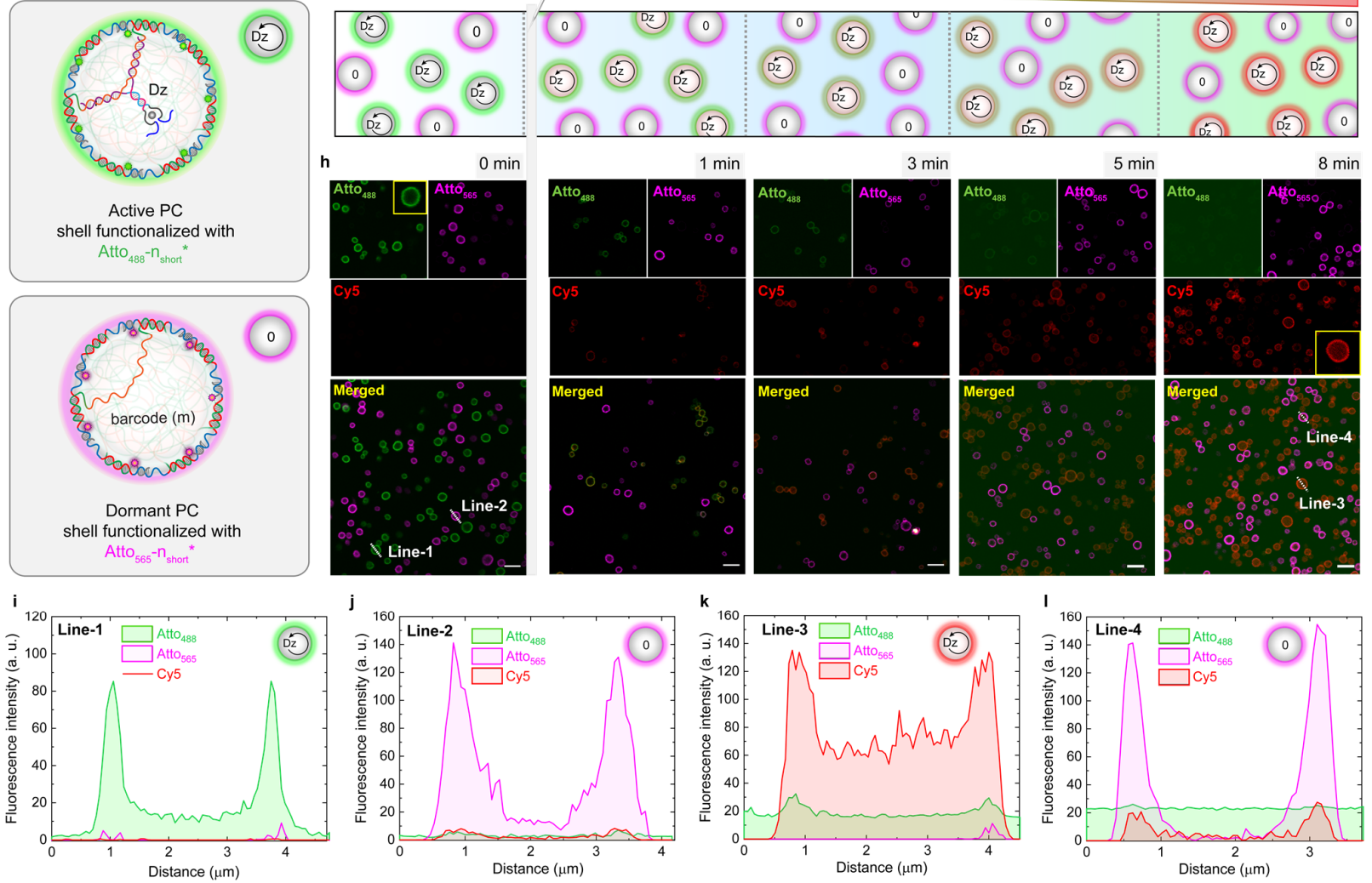

Figure 4. Intra-protocellular DNAzyme-catalyzed bond cleavage and ensuing downstream signal processing, leading to PC phenotype change. a) Schematic representation of the Dz-catalyzed RNA-linkage cleavage of Subs-3 and subsequent duplex melting, releasing the Cy5- $n *$ strand in situ, which triggers a downstream dynamic strand displacement reaction (DSD) at the shell of Atto $488-n_{\text {short }} *$ labeled DzCPCs. The cascade of events eventually changes the Shell fluorescence of PCs from green (Atto488) to red (Cy5). b) Schematic representation of two scenarios: 1) the DCBC and subsequent BCDM is performed in a buffered solution where the $\mathrm{Dz}$ is hybridized with a monomeric $m$ sequence and ii) $D C B C$ and $B C D M$ are performed in DzCPCs using Subs-3. c) The spectrofluorimetric analysis of DCBC and downstream BCDM in the case of Dz in solution (dark red trace) and at its encapsulated state (light red trace). The thermal uncaging of Subs-3 (in the absence of $\mathrm{Dz}$ ) is shown in a grey trace. d) and e) Time-dependent cytometric analysis of intra-protocellular DCBC, downstream BCDM, 
and DSD reaction via monitoring change in fluorescence intensity of Atto 488 . and Cy5 at each PC, respectively. The gradual decrease of Atto 488 fluorescence and rapid increase in Cy5 fluorescence indicates the metabolic phenotype change in DzCPCs. f) and g) Schematic representation of DCBC, downstream BCDM, and DSD reaction in a mixed PC system. The

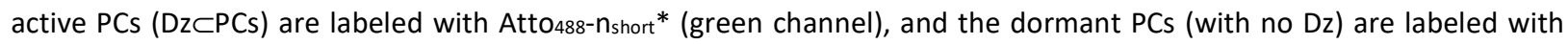
Att0565- $n_{\text {short }}$ * (magenta channel). h) Time-dependent CLSM images of metabolic phenotype change in the mixed PC system. At $t=0$ (before the addition of Subs-3), the active and dormant PCs are visualized as empty green and magenta. Upon Subs3 addition, a gradual increase of red fluorescence is observed at the shell of the active green PCs substituting the green fluorescence, while the magenta dormant PCs remain unchanged. i) and K) The line segment analysis on an active PC before (Line-1) and after (Line-3) the reaction and downstream transformation, respectively. j) and I) The line segment analysis on a dormant PC before (Line-2) and after (Line-4) the reaction and downstream transformation, respectively. Scale bars: 5

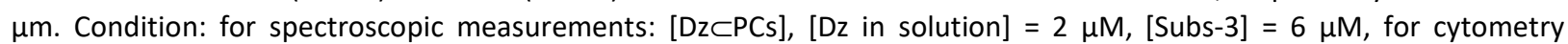
experiments: $[\mathrm{Dz} \subset \mathrm{PCs}]=0.5 \mu \mathrm{M},[$ Subs-1] $=1.5 \mu \mathrm{M}$ and for the microscopic experiments: $[\mathrm{Dz} \subset \mathrm{PCs}]=7 \mu \mathrm{M},[\mathrm{Subs}-1]=16.6$ $\mu \mathrm{M}$, at $37^{\circ} \mathrm{C}$, TE buffer at $\mathrm{pH} 8,50 \mathrm{mM} \mathrm{MgCl} 2$.

Moreover, we envisaged a more drastic downstream adaptation scenario by installing communication amongst active PCs via the metabolic presentation of an attractive multivalent interaction between them. In this context, we designed a substrate (Subs-4), which is similar to Subs-3 but features an additional palindromic sequence (6 $\mathrm{nt}=\mathrm{CTC}$ GAG) attached to Cy5-n* (Figure $5 \mathrm{a})$. The $T_{\mathrm{m}}$ of the palindrome $(\mathrm{p})$ is well below room temperature (17 $\left.{ }^{\circ} \mathrm{C}\right)$, so that Subs-4 does not form homodimers at the experimental temperature $\left(30^{\circ} \mathrm{C}\right)$. However, we expected that $p$ could induce inter-PC attraction by multivalent $p-p$ interactions. Indeed, upon the addition of Subs-4 into a dispersion of Atto $_{488}-\mathrm{n}_{\text {short }}{ }^{*}$-labeled DzCPCs, the shell color first changes over time from green to red due to the release of Cy5-n*-p and ensuing DSD at the shell. Critically, subsequently, the red PCs start forming "prototissue-like" aggregates arising from the formation of multivalent inter-protocellular p-p duplexes (Figure $5 \mathrm{~b}$-d). In a control experiment, when 10 equivalent (to the barcode $\mathrm{n}$ concentration) of Subs- 4 is added to a dormant PC (without the encapsulated Dz) suspension, no substantial PC crosslinking is observed (Supplementary Figure S7). These observations confirm that rationally designed signal transduction cascades combining catalytic Dz-based conversion, DSD reactions, and multivalent binding scenarios - allow establishing a metabolic PC system, in which a signal gets processed into an effector to establish phenotype changes and communication. This resembles, albeit on a simplistic level, adaptation pathways in living cellular communities. 


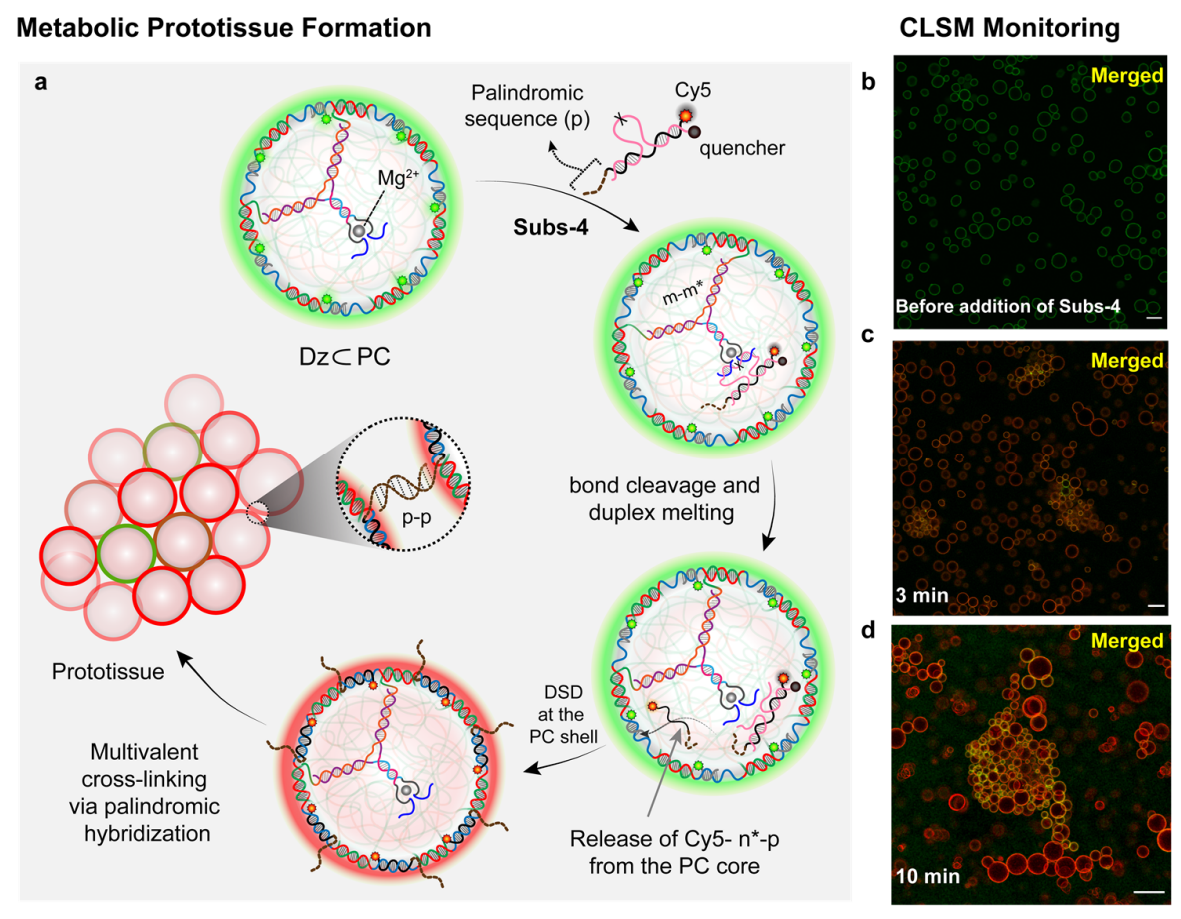

Figure 5. Metabolic bond-cleavage and downstream prototissue formation. a) Schematic representation of a downstream cascade, DCBC $\rightarrow$ BCDM $\rightarrow$ DSD reaction at PC shell $\rightarrow$ multivalent palindromic inter-protocellular duplex formation, after

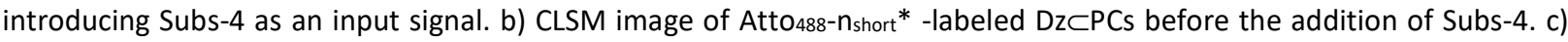
The CLSM image of DzCPCs after 3 min of substrate addition. The shell color starts changing to red as well as forming small $P C$ aggregates. d) At $t=8 \mathrm{~min}$, the CLSM image of a prototissue-like architecture built upon multivalent $p-p^{*}$ interactions at PC surface. The scale bar $=5 \mu \mathrm{m}$. The merged channel represents both Atto 488 (green) and Cy5 (red) fluorescence. Condition: $[\mathrm{Dz} \subset \mathrm{PCs}]=5 \mu \mathrm{M}$, [Subs-4] $=12.5 \mu \mathrm{M}$ in TE buffer at $\mathrm{pH} 8$ at $37^{\circ} \mathrm{C}$.

In summary, we introduced generic and versatile signal processing routines into communities of PCs by exploiting established DNAzyme and DSD reaction scenarios. The approach has the encapsulation of a DNAzyme inside the crowded macromolecular interior of a highly programmable all-DNA PC at its core. We then also demonstrated that the in-situ cleavage of an RNA-linkage could trigger several downstream processes that eventually alter the functional properties of the host PCs. This can be achieved on different levels of complexity by first targeting a phenotype change and subsequently inducing communication by multivalent interactions. These metabolic features are reminiscent of the functional behavior of a living cell at simplistic levels. We also revealed that the DNAzyme bond-cleaving activity is higher inside the crowded PCs. Even though DNAzymes have been established for several purposes, we believe that our approach to use this DNAzyme in a confined state and establish DNA-based downstream reaction cascade based on using the catalytic conversion to lead to PC adaptation will provide valuable insight into designing minimalistic life-like abiotic systems that can process, translate, communicate and relay DNA-based signals in more complex sensory environments. Taking into account recent advances in sensing antibodies,,$^{50}$ as well as the widely developed fields of DNA aptamers $5^{51}$ and DNAzymes, ${ }^{52}$ it appears feasible to achieve a broader sensing capability and to ultimately arrive at situations where communication between a natural cell and a PC can be established. 


\section{Acknowledgments}

We would like to thank Oliver S. Thomas for help with flow cytometry data analysis and plotting. A.S. acknowledges the support of the Alexander von Humboldt Foundation. We acknowledge the support by the European Research Council starting grant to A.W. (TimeProSAMat (agreement 677960)).

\section{Competing Interests}

The authors declare no competing interests

\section{Author contributions}

A. S. and A. W. conceived and designed the experiments. A. S. and M. H. performed the Cytometric experiments. A. S. performed the other experiments. W. L. optimized the palindromic sequences. A. S. analyzed the data and prepared the preliminary draft. A. W. and W. W. supervised the project. All the authors commented on the manuscript.

\section{References}

1. Pawson, T. \& Nash, P. Assembly of cell regulatory systems through protein interaction domains. Science 300, 445-452 (2003).

2. Pawson, T. Specificity in signal transduction: from phosphotyrosine-SH2 domain interactions to complex cellular systems. Cell 116, 191-203 (2004).

3. You, L., Cox, R.S., Weiss, R. \& Arnold, F.H. Programmed population control by cell-cell communication and regulated killing. Nature 428, 868-871 (2004).

4. Brangwynne, C.P. et al. Germline P granules are liquid droplets that localize by controlled dissolution/condensation. Science 324, 1729-1732 (2009).

5. Hyman, A.A., Weber, C.A. \& Juelicher, F. Liquid-liquid phase separation in biology. Annu. Rev. Cell Dev. Biol. 30, 39-58 (2014).

6. Buddingh', B.C. \& van Hest, J.C.M. Artificial Cells: Synthetic Compartments with Life-like Functionality and Adaptivity. Acc. Chem. Res. 50, 769-777 (2017).

7. Martin, N. Dynamic synthetic cells based on liquid-liquid phase separation. ChemBioChem 20, 25532568 (2019).

8. Szostak, J.W., Bartel, D.P. \& Luisi, P.L. Synthesizing life. Nature 409, 387-390 (2001).

9. Lyu, Y. et al. Protocells programmed through artificial reaction networks. Chem. Sci. 11, 631-642 (2020).

10. Zhu, T.F. \& Szostak, J.W. Coupled Growth and Division of Model Protocell Membranes. J. Am. Chem. Soc. 131, 5705-5713 (2009).

11. Adamala, K. \& Szostak, J.W. Nonenzymatic template-directed RNA synthesis inside model protocells. Science 342, 1098-1100 (2013).

12. Adamala, K. \& Szostak, J.W. Competition between model protocells driven by an encapsulated catalyst. Nat. Chem. 5, 495-501 (2013).

13. Kurihara, K. et al. Self-reproduction of supramolecular giant vesicles combined with the amplification of encapsulated DNA. Nat. Chem. 3, 775 (2011).

14. Adamala, K.P., Martin-Alarcon, D.A., Guthrie-Honea, K.R. \& Boyden, E.S. Engineering genetic circuit interactions within and between synthetic minimal cells. Nat. Chem. 9, 431-439 (2017).

15. Weiss, M. et al. Sequential bottom-up assembly of mechanically stabilized synthetic cells by microfluidics. Nat. Mater. 17, 89-96 (2018).

16. Peters, R.J.R.W. et al. Cascade Reactions in Multicompartmentalized Polymersomes. Angew. Chem. Int. Ed. 53, 146-150 (2014).

17. Che, H., Cao, S. \& van Hest, J.C.M. Feedback-Induced Temporal Control of "Breathing" Polymersomes To Create Self-Adaptive Nanoreactors. J. Am. Chem. Soc. 140, 5356 (2018). 
18. Dubuc, E. et al. Cell-free microcompartmentalised transcription-translation for the prototyping of synthetic communication networks. Curr. Opin. Biotechnol. 58, 72-80 (2019).

19. Sun, S. et al. Chemical Signaling and Functional Activation in Colloidosome-Based Protocells. Small 12, 1920-1927 (2016).

20. Tamate, R., Ueki, T. \& Yoshida, R. Evolved Colloidosomes Undergoing Cell-like Autonomous Shape Oscillations with Buckling. Angew. Chem. Int. Ed. 55, 5179-5183 (2016).

21. Joesaar, A. et al. DNA-based communication in populations of synthetic protocells. Nat. Nanotechnol. 14, 369-378 (2019).

22. Silverman, A.D., Karim, A.S. \& Jewett, M.C. Cell-free gene expression: an expanded repertoire of applications. Nat. Rev. Genet. 21, 151-170 (2020).

23. Yewdall, N.A., André, A.A.M., Lu, T. \& Spruijt, E. Coacervates as models of membraneless organelles. Curr. Opin. Colloid Interface Sci., 101416 (2020).

24. Deshpande, S. \& Dekker, C. Studying phase separation in confinement. Curr. Opin. Colloid Interface Sci . 52, 101419 (2021).

25. Dora Tang, T.Y. et al. Fatty acid membrane assembly on coacervate microdroplets as a step towards a hybrid protocell model. Nat. Chem. 6, 527-533 (2014).

26. Deng, J. \& Walther, A. Programmable ATP-Fueled DNA Coacervates by Transient Liquid-Liquid Phase Separation. Chem 6, 3329-3343 (2020).

27. Goodsell, D.S. Inside a living cell. Trends Biochem. Sci. 16, 203-206 (1991).

28. Fulton, A.B. How crowded is the cytoplasm? Cell 30, 345-347 (1982).

29. Ellis, R.J. Macromolecular crowding: obvious but underappreciated. Trends Biochem. Sci. 26, 597-604 (2001).

30. Göpfrich, K., Platzman, I. \& Spatz, J.P. Mastering Complexity: Towards Bottom-up Construction of Multifunctional Eukaryotic Synthetic Cells. Trends Biotechnol. 36, 938-951 (2018).

31. Sokolova, E. et al. Enhanced transcription rates in membrane-free protocells formed by coacervation of cell lysate. Proc. Natl. Acad. Sci. U.S.A. 110, 11692-11697 (2013).

32. Strulson, C.A., Molden, R.C., Keating, C.D. \& Bevilacqua, P.C. RNA catalysis through compartmentalization. Nat. Chem. 4, 941-946 (2012).

33. Poudyal, R.R. et al. Template-directed RNA polymerization and enhanced ribozyme catalysis inside membraneless compartments formed by coacervates. Nat. Commun. 10, 490 (2019).

34. Crosby, J. et al. Stabilization and enhanced reactivity of actinorhodin polyketide synthase minimal complex in polymer-nucleotide coacervate droplets. Chem. Commun. 48, 11832-11834 (2012).

35. Booth, R., Qiao, Y., Li, M. \& Mann, S. Spatial positioning and chemical coupling in coacervate-inproteinosome protocells. Angew. Chem. Int. Ed. 58, 9120-9124 (2019).

36. Merindol, R., Loescher, S., Samanta, A. \& Walther, A. Pathway-controlled formation of mesostructured all-DNA colloids and superstructures. Nat. Nanotechnol. 13, 730-738 (2018).

37. Samanta, A., Sabatino, V., Ward, T.R. \& Walther, A. Functional and morphological adaptation in DNA protocells via signal processing prompted by artificial metalloenzymes. Nat. Nanotechnol. 15, 914-921 (2020).

38. Qian, L., Winfree, E. \& Bruck, J. Neural network computation with DNA strand displacement cascades. Nature 475, 368-372 (2011).

39. Chirieleison, S.M., Allen, P.B., Simpson, Z.B., Ellington, A.D. \& Chen, X. Pattern transformation with DNA circuits. Nat. Chem. 5, 1000-1005 (2013).

40. Gerasimova, Y.V. \& Kolpashchikov, D.M. Towards a DNA Nanoprocessor: Reusable Tile-Integrated DNA Circuits. Angew. Chem. Int. Ed. 55, 10244-10247 (2016).

41. Heinen, L. \& Walther, A. Programmable dynamic steady states in ATP-driven nonequilibrium DNA systems. Sci. Adv. 5, eaaw0590 (2019).

42. Deng, J. \& Walther, A. Fuel-Driven Transient DNA Strand Displacement Circuitry with Self-Resetting Function. J. Am. Chem. Soc. 142, 21102-21109 (2020).

43. Zhang, D.Y. \& Seelig, G. Dynamic DNA nanotechnology using strand-displacement reactions. Nat. Chem. 3, 103 (2011).

44. Elbaz, J. et al. DNA computing circuits using libraries of DNAzyme subunits. Nat. Nanotechnol. 5, 417422 (2010). 
45. Tang, L. et al. Colorimetric and Ultrasensitive Bioassay Based on a Dual-Amplification System Using Aptamer and DNAzyme. Analytical Chemistry 84, 4711-4717 (2012).

46. Liu, S., Cheng, C., Gong, H. \& Wang, L. Programmable Mg2+-dependent DNAzyme switch by the catalytic hairpin DNA assembly for dual-signal amplification toward homogeneous analysis of protein and DNA. Chem. Commun. 51, 7364-7367 (2015).

47. Gong, L. et al. DNAzyme-based biosensors and nanodevices. Chem. Commun. 51, 979-995 (2015).

48. Elbaz, J., Wang, F., Remacle, F. \& Willner, I. pH-Programmable DNA Logic Arrays Powered by Modular DNAzyme Libraries. Nano Lett. 12, 6049-6054 (2012).

49. Ludwanowski, S., Samanta, A., Loescher, S., Barner-Kowollik, C. \& Walther, A. A Modular Fluorescent Probe for Viscosity and Polarity Sensing in DNA Hybrid Mesostructures. Adv. Sci. 8, 2003740 (2021).

50. Baranda Pellejero, L. et al. Using antibodies to control DNA-templated chemical reactions. Nat. Commun. 11, 6242 (2020).

51. $\mathrm{Ni}$, S. et al. Recent Progress in Aptamer Discoveries and Modifications for Therapeutic Applications. ACS Appl. Mater. Interfaces 13, 9500-9519 (2021).

52. Zhang, H.Q., Li, F., Dever, B., Li, X.F. \& Le, X.C. DNA-Mediated Homogeneous Binding Assays for Nucleic Acids and Proteins. Chem. Rev. 113, 2812-2841 (2013). 